\title{
Bei Arbeitsunfällen gibt es deutlich mehr Honorar
}

\begin{abstract}
— Von 2017-2020 werden die Gebührensätze der UV-GOÄ jeweils zum 1. Oktober 2017 erhöht. In diesem Jahr geht es um $8 \%$ nach oben, in den drei folgenden Jahren um jeweils 3\%. Die Erhöhung erfolgt basiswirksam, sodass sich
\end{abstract}

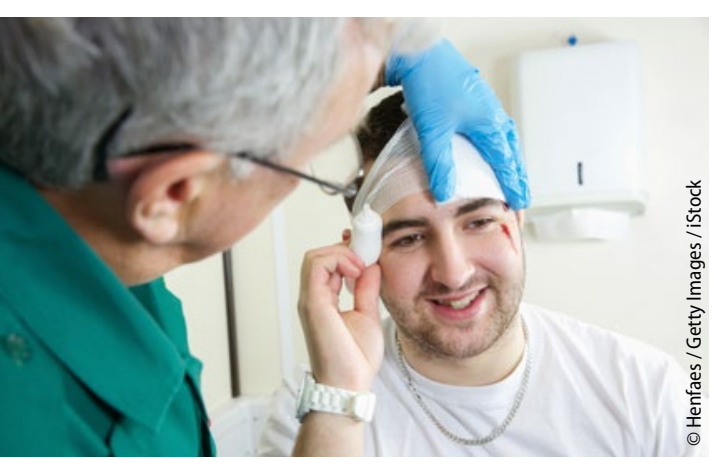

Erstversorgung eines Arbeitsunfalls. insgesamt eine Steigerung von rund 18\% ergibt. Dieses deutliche Plus kommt allen Vertragsärzten zugute, die für die Unfallversicherung tätig sind - also auch Hausärzten.

Zusätzlich gibt es zum 1. April 2018 einige Neuerungen bei den Formularen. Im hausärztlichen Bereich ist beachtenswert, dass für Überweisungen nach den $\$ \$ 26,39$ und 41 des Vertrags Ärzte/Unfallversicherungsträger - also auch für die Überweisung an den D-Arzt - die Nr. 145 UV-GOÄ abgerechnet werden kann und der bisherige Vordruck F 2900 entfällt. Die aktualisierte UV-GOÄ gibt es unter www.kbv.de/html/93.php.

\section{MMW-KOMMENTAR}

Bei einem Arbeitsunfall muss eine ärztliche Unfallmeldung nach Muster F 1050 sofort

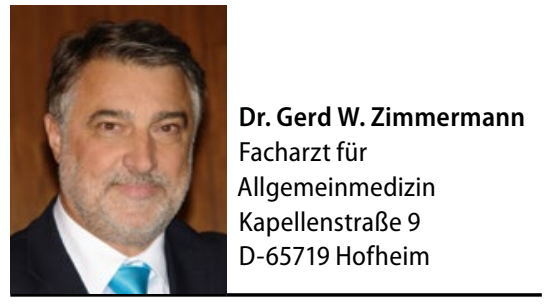

am Unfalltag, spätestens am Folgetag erstellt und an die zuständige Berufsgenossenschaft (BG) verschickt werden. Dies kann mit der Nr. 125 UV-GOÄ berechnet werden, ab Oktober für 8,10 Euro. Zusätzlich ist die Berechnung aller Leistungen nach UV-GÖ̈ möglich, die bei einer Erstversorgung notwendig sind, etwa Beratung, Untersuchung, Wundversorgung oder Verbände. Wird der Patient direkt an den D-Arzt weitergeleitet, kommt nur die Nr. 145 UV-GOÄ zum Ansatz. Im Oktober steigt ihre Bewertung auf 3,77 Euro. Der D-Arzt kann den Patienten dann wieder in den Rahmen der Allgemeinen Heilbehandlung überweisen, etwa an den Hausarzt.

Für die Meldung einer Berufskrankheit wird der Vordruck F 6000 verwendet. Abgerechnet wird mit der Nr. 141 UV-GOÄ, die ab Oktober mit 16,44 Euro bewertet ist.

\section{Arzneimittelsoftware hinkt gen Zukunft}

_ Bereits 2006 hat der Gesetzgeber vorgegeben, dass in Arztpraxen nur Softwareprodukte genutzt werden dürfen, die eine manipulationsfreie Verordnung der Arzneimittel gewährleisten. Die Software muss auch einschlägige, für den Arzt relevante Informationen enthalten, etwa die Regelungen der Arzneimittelrichtlinie. Geprüft und zertifiziert wird die Arzneiverordnungssoftware von der KBV.

Nun muss der Anforderungskatalog angepasst werden, v. a. aufgrund der Neuregelungen aus dem Gesetz für die sichere digitale Kommunikation und Anwendungen im Gesundheitswesen vom Dezember 2015, dem sogenannten E-Health-Gesetz.

\section{MMW-KOMMENTAR}

Das Gesetz gibt vor, dass Vertragsärzte für die Verordnung nur solche Programme nutzen dürfen, bei denen die Informationen zu Arzneimitteln - etwa die Preise oder die Inhalte der Richtlinie - auf dem aktuellen Stand sind. KBV und GKV-Spitzenverband haben sich zunächst nicht einigen können, unter Vermittlung des Bundesschiedsamts aber verabredet, dass ab 1. April 2018 die Aktualisierung der Arzneimittelstammdaten innerhalb der Verordnungssoftware mindestens monatlich erfolgen soll.

Es gibt auch Argumente für einen kürzeren Zyklus: In der Gesetzesbegründung wird festgestellt, dass die in den Apotheken zur Abrechnung verwendeten Programme alle 14 Tage ihre Inhalte aktualisieren müssen. Ein solcher Zyklus wird allerdings erst verpflichtend, wenn die Softwareanbieter eine standardisierte Schnittstelle eingeführt haben, die den Vertragsärzten den Wechsel der Verordnungssoftware bzw. der Arzneimitteldatenbanken erleichtern soll. Dies wird wohl ab Juli 2020 der Fall sein.

Die KBV hat in diesem Zusammenhang gefordert, dass künftig die Krankenkassen die Kosten übernehmen, wenn aufgrund neuer gesetzlicher Vorgaben Mehrkosten für die Praxissoftware entstehen. Dies soll auch im Bundesmantelvertrag-Ärzte festgehalten werden. Die KBV verweist dabei auch auf die Einführung des bundeseinheitlichen Medikationsplans. Mit den Kassen konnte eine Einigung in dieser Sache allerdings bisher nicht erzielt werden. 